Pacific Journal of Mathematics

LOWER BOUNDS FOR HIGHER EIGENVALUES BY FINIT 


\title{
LOWER BOUNDS FOR HIGGHER EIGENVALUES BY FINITE DIFFERENCE METHODS
}

\author{
H. F. WEINBERGER
}

1. Introduction. This paper gives lower bounds for all the eigenvalues of an arbitrary second order self-adjoint elliptic differential operator on a bounded domain $R$ with zero boundary conditions in terms of the eigenvalues of an associated finite difference problem. When $R$ is sufficiently smooth, the lower bounds converge to the eigenvalues themselves as the mesh size approaches zero. A certain class of selfabjoint systems of elliptic differential equations containing no mixed derivatives is also treated.

Upper bounds for the eigenvalues of a differential operator can always be found by the Rayleigh-Ritz method. That is, one puts piecewise differentiable functions vanishing on the boundary into the Poincare inequality [14]. It was pointed out by Courant [2] that in the case of second order operators one can reduce the problem of upper bounds to a finite difference eigenvalue problem by using piecewise linear functions (see $\S 6$ ).

Lower bounds are more difficult to find. The only known method giving arbitrarily close lower bounds for the eigenvalues is that of $A$. Weinstein [20], which is usually quite difficult to apply. It was shown by G. E. Forsythe $[5,6,7]$ that if the eigenvalues $\lambda_{1} \leqq \lambda_{2} \leqq \cdots$ of the two-dimensional problem

$$
\Delta u+\lambda u=0 \quad \text { in } R
$$

with $u=0$ on the boundary are approximated by the eigenvalues $\lambda_{1}^{(h)} \leqq \lambda_{2}^{(h)} \leqq \cdots$ of a certain finite difference problem on a mesh of size $h$, then there exist constants $\gamma_{,}^{(1)} \gamma_{,}^{(2)} \ldots$ such that

$$
\lambda_{k}^{(h)} \leqq \lambda_{k}-\gamma^{(k)} h^{2}+o\left(h^{2}\right) \text {. }
$$

The $\gamma^{(k)}$ cannot be computed, but are positive for convex $R$. However, the $o\left(h^{2}\right)$ term is completely unknown, so that this asymptotic formula cannot be used to bound $\lambda_{k}$ below.

It was shown independently by J. Hersch [8] and the author [18, 19] that if $\lambda_{1}$ is the lowest eigenvalue of (1.1) and if $\lambda_{1}^{(h)}$ is the lowest eigenvalue of a finite difference problem on a mesh that is slightly arger than $R$, then $\lambda_{1}^{(h)}$ and, in fact, a quantity slightly larger than $\lambda_{1}^{(h)}$ are lower bounds for $\lambda_{1}$.

Received September 17, 1957. Sponsored by the United States Army under Contract No. DA-11-022-ORD-2059. 
This result is here extended to higher eigenvalues, higher dimensions, and variable coefficients by a modification of the method previously used by the author. The basic idea is to define a mesh function by an average over mesh squares of a linear combination of the first $k$ eigenfunctions of (1.1). One then defines the finite difference eigenvalue problem in such a way that its Rayleigh quotient evaluated for this mesh function can be estimated in terms of the unknown eigenvalue $\lambda_{k}$. By the Poincare inequality this leads to an upper bound for the eigenvalue $\lambda_{k}^{(h)}$ in terms of $\lambda_{k}$, which serves as a lower bound for $\lambda_{k}$ in terms of $\lambda_{k}^{(h)}$. ${ }^{1}$

For the sake of clarity, the method is first presented for the problem (1.1) in $\S 2$. It must be noted that while the lower bound (2.25) holds for all $\lambda_{k}$, it is not as good for $\lambda_{1}$ as the bound previously given either by Hersch [8] or the author [19]. It is smaller, rather than larger, than $\lambda_{1}^{(h)}$ by a term of order $h^{2}$.

The method extends easily to an equation in $N$ dimensions with variable coefficients when the operator contains no mixed derivatives. This extension is made in $\S 3$. Again the lower bound is smaller than $\lambda_{k}^{(h)}$ by a term of order $h^{2}$.

In $\S 4$ the general second order self-adjoint operator is considered. The presence of mixed derivatives introduces complications. The lower bound becomes $\lambda_{k}^{(h)}$ reduced by a term of order $h^{1 / 2}$. Furthermore, it becomes necssary to assume that $R$ has no re-entrant cusps, corners, or edges, and that it does not have infinite oscillations.

Section 5 presents an extension of the lower bound to a self-adjoint system of second order equations with no mixed derivatives. The extension to a system with mixed derivatives appears to be very difficult, and is not done.

In $\S 6$ the difference between upper and lower bound is discussed. It is estimated explicitly for convex $R$. At the same time this discussion serves to show when the lower bounds converge to the eigenvalues.

In $\S 7$ we take account of the fact that the solution vanishing on the boundary of a non-homogeneous differential equation can be characterized by a minimum principle (Dirichlet's principle). Using the methods developed for eigenvalues, we give a method for finding a lower bound for this minimum. It is, of course, true that in this case one can get a get a lower bound by Thomson's principle. However, this principle involves solutions of the differential equation which may be difficult to find as well as difficult to compute with. Finite difference methods are more amenable to high speed computation. The upper and lower bounds so obtained, together with the function that gives the upper bound, can be used to find upper and lower bounds for the solution at an $\left|\lambda_{k}^{(h)}-\lambda_{k}\right|$. 
interior point by the method of Diaz and Greenberg [3, 4].

Section 8 indicates the extension of our method to an important class of higher order operators. This extension is applied to the problem of the vibrating clamped plate.

2. The basic bound. Let the eigenvalues of

$$
\begin{array}{ll}
\Delta u+\lambda u=0 & \text { in } R, \\
u=0 & \text { on the boundary } \dot{R}
\end{array}
$$

be denoted by

$$
\lambda_{1} \leqq \lambda_{2} \leqq \cdots
$$

Let the corresponding eigenfunctions, normalized so that

$$
\int_{R} u^{2} d x d y=1
$$

be denoted by $u_{1}, u_{2}, \cdots$.

Consider the $x-y$ plane divided into squares by lines $x=m h, y=n h$, $m, n=0, \pm 1, \pm 2 \cdots$. Let $R_{h}$ be a region consisting of a union of entire squares of this grid and having the property of containing not only $R$, but also all its left and downward translates of distances up to $h:^{2}$

$$
R_{h} \supset\{(x, y) \mid(x+\alpha, y+\beta) \in R \text { for some } 0 \leqq \alpha \leqq h, 0 \leqq \beta \leqq h\}
$$

We consider the class $M_{h}$ of functions $v(m h, n h)$ defined at mesh points $(m h, n h)$ in $R_{h}$ and vanishing at boundary points of $R_{h}$. The eigenvalues (2.2) are to be approximated by the eigenvalues

$$
\lambda_{1}^{(h)} \leqq \lambda_{2}^{(h)} \leqq \cdots
$$

of the finite difference problem

$$
\Delta_{h} v+\lambda^{(h)} v=0
$$

where $v$ is a mesh function of the class $M_{h}$, and

$$
\begin{aligned}
\Delta_{h} v= & h^{-2}[v(m h+h, n h)+v(m h-h, n h)+v(m h, n h+h) \\
& +v(m h, n h-h)-4 v(m h, n h)] .
\end{aligned}
$$

The eigenvalues (2.5) are bounded above by the Poincare (RayleighRitz) inequality [14], which states that for $v_{1}, v_{2}, \cdots, v_{k}$ of class $M_{h}$ and linearly independent

2 Equivalently, if the intersection of $R$ and the square $m h<x<(m+1) h, n h<y<(n+1) h$ is non-empty, then $(m h, n h)$ is an interior point of $R_{h}$. 


$$
\lambda_{k}^{(h)} \max _{\xi_{1}, \cdots, \xi_{k}} \frac{D_{h}\left(\xi_{1} v_{1}+\cdots+\xi_{k} v_{k}\right)}{h^{2} \sum_{(m h, n h) \in R_{h}}\left(\xi_{1} v_{1}+\cdots+\xi_{k} v_{k}\right)^{2}},
$$

where

$$
D_{h}(v) \underset{(m h, n h) \in R_{h}}{\equiv}\left\{[v(m h+h, n h)-v(m h, n h)]^{2}+[v(m h, n h+h)-v(m h, n h)]^{2}\right\}
$$

Let $u(x, y)$ be a continuous piecewise continuously differentiable function in the whole $x-y$ plane which vanishes outside $R$. We define the mesh function

$$
v(m h, n h)=h^{-2} \int_{0}^{h} \int_{0}^{h} u(m h+\alpha, n h+\beta) d \alpha d \beta .
$$

Because of (2.4) this function belongs to $M_{h}$. We note that

$$
\begin{aligned}
& \int_{R} \int^{2} d x d y-\underset{(m h, n h) \in R_{h}}{h^{2}} \underset{(m h, n h)^{2}}{ } \\
& \underset{(m h,, n h) \in R_{h}}{=} \int_{0}^{h} \int_{0}^{h}[u(m h+\alpha, n h+\beta)-v(m h, n h)]^{2} d \alpha d \beta .
\end{aligned}
$$

By definition (2.10)

$$
\int_{0}^{h} \int_{0}^{h}[u(m h+\alpha, n h+\beta)-v(m h, n h)] d \alpha d \beta=0 .
$$

Consequently, each integral on the right of (2.11) is bounded by the integral of the gradient of $u$ times the reciprocal of the second free membrane eigenvalue for the square of side $h$ :

$$
\begin{aligned}
& \int_{0}^{h} \int_{0}^{h}[u(m h+\alpha, n h+\beta)-v(m h, n h)]^{2} d \alpha d \beta \\
\leqq & \frac{h^{2}}{\pi^{2}} \int_{0}^{h} \int_{0}^{h}|\operatorname{grad} u(m h+\alpha, n h+\beta)|^{2} d \alpha d \beta .
\end{aligned}
$$

Replacing this in (2.11) and summing over all the squares, we have

$$
\iint_{R} u^{2} d x d y-h^{2} \sum_{R_{h}} v^{2} \leqq \frac{h^{2}}{\pi^{2}} \iint_{R}|\operatorname{grad} u|^{2} d x d y
$$

Now let

$$
u=\xi_{1} u_{1}+\cdots+\xi_{k} u_{k},
$$

where the $u_{i}$ are the normalized eigenfunctions of (2.1), and the $\xi_{i}$ are any real numbers. Then we have

$$
v=\xi_{1} v_{1}+\cdots+\xi_{k} v_{k}
$$


where the $v_{i}$ are defined in terms of the $u_{i}$ just as $v$ is defined in terms of $u$ by (2.10). Inequality (2.14) can be written in the form

$$
h^{2} \sum_{R_{h}}\left(\xi_{1} v_{1}+\cdots+\xi_{k} v_{k}\right)^{2} \geqq \sum_{i=1}^{k} \xi_{i}^{2}-\frac{h^{2}}{\Pi^{2}} \sum_{i=1}^{k} \lambda_{i} \xi_{i}^{2} .
$$

This gives a lower bound for the denominator of the ratio in (2.8). In order to be certain that the mesh functions $v_{i}$ are linearly independent, we assume that $h$ is chosen so small that this lower bound is always positive. That is, we take

$$
h^{2}<\pi^{2} / \lambda_{k}
$$

We now turn to the numerator in (2.8). We note that if $u$ and $v$ are again related by $(2.10)$, we have

$$
\begin{aligned}
& v(m h+h, n h)-v(m h, n h) \\
= & h^{-2} \int_{0}^{2 h} d \alpha \int_{0}^{h} d \beta \psi(\alpha) \frac{\partial u}{\partial x}(m h+\alpha, n h+\beta),
\end{aligned}
$$

with a similar formula for $v(m h, n h+h)-v(m h, n h)$. Here we have put

$$
\psi(\alpha)= \begin{cases}\alpha & 0 \leqq \alpha \leqq h \\ 2 h-\alpha & h \leqq \alpha \leqq 2 h \\ 0 & \text { elsewhere }\end{cases}
$$

so that

$$
\psi(\alpha)+\psi(\alpha+h)+\psi(\alpha-h)=h, \int_{0}^{2 h} \psi(\alpha) d \alpha=h^{2} .
$$

Consequently, we can write

$$
\begin{aligned}
& \iint_{R}|\operatorname{grad} u|^{2} d x d y-D_{h}(v) \\
= & h^{-1} \sum_{R_{h}} \int_{0}^{2 h} d \alpha \int_{0}^{h} d \beta \psi(\alpha)\left[\left\{\frac{\partial u}{\partial x}(m h+\alpha, n h+\beta)\right.\right. \\
- & \left.h^{-1}\langle v(m h+h, n h)-v(m h, n h)\rangle\right\}^{2} \\
+ & \left.\left\{\frac{\partial u}{\partial y}(m h+\beta, n h+\alpha)-h^{-1}\langle v(m h, n h+h)-v(m h, n h)\rangle\right\}^{2}\right] \geqq 0 .
\end{aligned}
$$

Again making the substitutions (2.15) and (2.16), we have

$$
D_{h}\left(\xi_{1} v_{1}+\cdots+\xi_{k} v_{k}\right) \leqq \sum_{i=1}^{k} \lambda_{i} \xi_{i}^{2} .
$$

Inserting $(2,17)$ and $(2.23)$ in the bound $(2.8)$ yields 


$$
\lambda_{k}^{(h)} \leqq \max _{\xi_{1}, \ldots, \xi_{k}} \frac{\sum_{i=1}^{k} \lambda_{i} \xi_{i}^{2}}{\sum_{i=1}^{k}\left(1-\frac{h^{2} \lambda_{i}}{\pi^{2}}\right) \xi_{i}^{2}}=\frac{\lambda_{k}}{1-\frac{h^{2} \lambda_{k}}{\pi^{2}}} .
$$

Solving for $\lambda_{k}$ we find the lower bound

$$
\lambda_{k} \geqq \frac{\lambda_{k}^{(h)}}{1+\frac{h^{2} \lambda_{k}^{(h)}}{\pi^{2}}} \cdot
$$

This bound was derived under the assumption that (2.18) holds. However, if (2.18) is violated, (2.25) is trivially true. Thus, the lower bound (2.25) holds for all $k$ such that $\lambda_{k}^{(h)}$ is defined ( $k$ at most equal to the number of interior mesh points of $R_{h}$ ). The same type of consideration will apply in all the derivations to follow. That is, one derives the lower bound by assuming an inequality like (2.18) to hold, and then finds that the lower bound also holds when the inequality is violated. We shall suppress this argument in what follows.

3. Variable coefficients, no mixed derivatives. We now extend the results of the preceding section to an eigenvalue problem in $N$ dimensions. We consider the problem

$$
\begin{aligned}
& -\sum_{i=1}^{N} \frac{\partial}{\partial x^{i}}\left(p^{i} \frac{\partial u}{\partial x^{i}}\right)+q u=\lambda r u \text { in } R, \\
& u=0 \quad \text { on the boundary } \dot{R} .
\end{aligned}
$$

Here $R$ is a bounded $N$-dimensional domain. The functions $p^{i}, q$, and $r$ are assumed to be piecewise continuously differentiable. We assume $p^{i}$ and $r$ to be positive and $q$ non-negative in the closure of $R$. The eigenvalues are arranged in increasing order

$$
\lambda_{1} \leqq \lambda_{2} \leqq \cdots
$$

and the corresponding eigenfunctions, normalized by

$$
\int_{R} r u^{2} d v=1
$$

are called $u_{1}, u_{2}, \cdots$.

The space is divided into $N$-cubes by the planes $x^{i}=m^{i} h, m^{i}=0$, $\pm 1, \pm 2, \cdots$.

We again denote by $R_{h}$ a region consisting of the union of mesh cubes, and containing not only $R$ but all its translates in negative $x$-directions of distances up to $h$. We denote by $M_{h}$ the class of functions 
$v\left(m^{1} h, \cdots, m^{N} h\right)$ defined at mesh points and vanishing at all such points on the bounday of or exterior to $R_{h}$.

Let $u\left(x^{1}, \cdots, x^{N}\right)$ be a continuous piecewise differentiable function vanishing outside $R$. Then by definition of $R_{h}$ the mesh function

$$
v\left(m^{1} h, \cdots, m^{N} h\right)=h^{-N} \int_{0 \leqq \alpha^{i} \leqq h} u\left(m^{i} h+\alpha^{i}\right) d \alpha^{1} \cdots d \alpha^{N}
$$

is in $M_{h}$. We define the mesh function ${ }^{3}$

$$
\bar{r}\left(m^{i} h\right)=\left[h^{-N} \int_{0 \leqq \alpha^{i} \leqq h} \frac{d \alpha^{1} \cdots d \alpha^{N}}{r\left(m^{i} h+\alpha^{i}\right)}\right]^{-1} .
$$

Analogous to (2.11) we have the identity

$$
\begin{aligned}
& \int_{R} r u^{2} d V-h^{N} \sum \bar{r}\left(m^{i} h\right) v\left(m^{i} h\right)^{2} \\
= & \sum_{R_{h}} \int_{0 \in \alpha^{i} \leqq h}\left[r\left(m^{i} h+\alpha^{i}\right) u\left(m^{i} h+\alpha^{i}\right)-\bar{r}\left(m^{i} h\right) v\left(m^{i} h\right)\right]^{2} \frac{d \alpha^{1} \cdots d \alpha^{N}}{r\left(m^{i} h+\alpha^{i}\right)} .
\end{aligned}
$$

Also, by (3.4) and (3.5)

$$
\int_{0 \leqq \alpha^{i} \leqq h}\left[r\left(m^{i} h+\alpha^{i}\right) u\left(m^{i} h+\alpha^{i}\right)-\bar{r}\left(m^{i} h\right) v\left(m^{i} h\right)\right] \frac{d \alpha^{1} \cdots d \alpha^{N}}{r\left(m^{i} h+\alpha^{i}\right)}=0 .
$$

Thus, we are again led to a free membrane problem, and we find

(3.8) $\int_{0 \leqq \alpha^{i} \leqq h}[r u-\bar{r} v]^{2} \frac{d \alpha^{1} \cdots d \alpha^{N}}{r} \leqq \frac{h^{2}}{\pi^{2} r_{m}} \int_{0 \leqq \alpha^{i} \leqq n}\left|\operatorname{grad} r u\left(m^{i} h+\alpha^{i}\right)\right|^{2} d \alpha^{1} \cdots d \alpha^{N}$,

where we have put

$$
r_{m}=\min _{x \in R} r\left(x^{1}, \cdots, x^{N}\right)
$$

By the triangle inequality

$$
\left\{\int_{R}|\operatorname{grad} r u|^{2} d V\right\}^{1 / 2} \leqq\left\{\int_{R} r^{2}|\operatorname{grad} u|^{2} d V\right\}^{1 / 2}+\left\{\int_{R} u^{2}|\operatorname{grad} r|^{2} d V\right\}^{1 / 2}
$$

\section{Hence we have}

3 The definition of $r(x)$ outside $R$ is rather arbitrary. We choose it in such a way that the term in the bracket is the mean value of $r$ over the intersection of the domain of integration with $R$. Since $\lambda_{k}^{(h)}$ decreases with increasing $R_{h}$, we can assume without loss of generality that $R_{h}$ is minimal with respect to the analogue of $(2.4)$, so that for squares corresponding to interior points of $R_{h}$ this intersection is not empty. Similar considerations will apply to the mesh functions formed from the other coefficients. 


$$
\begin{gathered}
h^{N} \sum_{R_{h}} \bar{r} v^{2} \geqq \int_{R} r u^{2} d V-\frac{h^{2}}{\pi^{2} r_{m}}\left[K \int _ { R } \left[p^{1}\left(K \frac{\partial u}{\partial x^{1}}\right)^{2}+\cdots\right.\right. \\
\left.\left.\left.+p^{N}\left(\frac{\partial u}{\partial x^{N}}\right)^{2}+q u^{2}\right] d V\right\}^{1 / 2}+\left\{L \int_{R} r u^{2} d V\right\}^{1 / 2}\right]^{2}
\end{gathered}
$$

where

$$
\begin{aligned}
& K=\max _{\substack{x \in R \\
i=1, \cdots, N}}\left(\frac{r^{2}}{p^{i}}\right), \\
& L=\max \left(\frac{|\operatorname{grad} r|^{2}}{r}\right) .
\end{aligned}
$$

We also find

$$
\int_{R} q u^{2} d V-h^{N} \sum_{R_{h}} \bar{q} v^{2}=\sum_{R_{h}} \int_{0 \leqq \alpha^{i} \leqq h}[q u-\overline{q v}]^{2} \frac{d \alpha^{1} \cdots d \alpha^{N}}{q} \geqq 0,
$$

where we have put ${ }^{4}$

$$
\bar{q}\left(m^{i} h\right)=\left[h^{-N} \int_{0 \leqq \alpha^{i} \leqq h} \frac{d \alpha^{1} \cdots d \alpha^{N}}{q\left(m^{i} h+\alpha^{i}\right)}\right]^{-1} .
$$

Using the function $\psi(\alpha)$ defined by (2.20), we find that

$$
\begin{aligned}
& \int_{R} p^{1}\left(\frac{\partial u}{\partial x^{1}}\right)^{2} d V-h^{N-2} \sum_{R_{h}} \overline{p^{1}}\left(m^{i} h\right)\left[v\left(m^{1} h+h, m^{2} h, \cdots, m^{N} h\right)\right. \\
& \left.-v\left(m^{1} h, \cdots, m^{N} h\right)\right]^{2}=h^{-1} \sum_{R_{h}} \int_{\substack{0 \leq \alpha_{1} \leq 2 h \\
0 \leqq \infty_{j} \leq h, j>1}}\left\{p^{1}\left(m^{i} h+\alpha^{i}\right) \frac{\partial u}{\partial x^{1}}\left(m^{i} h+\alpha^{i}\right)\right. \\
& \left.-h^{-1} \overline{p^{1}}\left[v\left(m^{1} h+h\right)-v\left(m^{1} h\right)\right]\right\}^{2} \psi\left(\alpha^{1}\right) \frac{d \alpha^{1} \cdots d \alpha^{N}}{p^{1}\left(m^{i} h+\alpha^{i}\right)} \geqq 0,
\end{aligned}
$$

where we have put ${ }^{3}$

$$
\overline{p^{1}}\left(m^{i} h\right)=\left[h^{-N-1} \int_{\substack{0 \leq \alpha_{1} \leq 2 h \\ 0 \leqq \alpha_{j} \leq n, j>1}} \frac{\psi\left(\alpha^{1}\right) d \alpha^{1} \cdots d \alpha^{N}}{p^{1}\left(m^{i} h+\alpha^{i}\right)}\right]^{-1} .
$$

In this way we find that if we define the quadratic form

$$
Q(w)=h^{N} \sum_{R_{h}}\left\{h^{-2} \sum_{j=1}^{N} \overline{p^{j}}\left[w\left(m^{i} h+\delta_{i j} h\right)-w\left(m^{i} h\right)\right]^{2}+\bar{q} w^{2}\right\}
$$

for mesh functions $w$ in $M_{h}$, where ${ }^{3}$

$$
\bar{p}^{j}=\left[h^{-N-1} \int_{\substack{0 \leqq \alpha^{j} \leqq 2 h \\ 0 \leqq \alpha^{l} \leqq h, l \neq j}} \frac{\varphi\left(\alpha^{j}\right) d \alpha^{1} \cdots d \alpha^{N}}{p^{j}\left(m^{i} h+\alpha^{i}\right)}\right]^{-1}
$$

${ }^{4}$ See footnote 3 . We make the convention that $\bar{q}=0$ if the integral diverges or if $q=0$ in an open set. 
and $\bar{q}$ is defined by (3.14), then

$$
Q(v) \leqq \int_{R}\left[p^{1}\left(\frac{\partial u}{\partial x^{1}}\right)^{2}+\cdots+p^{N}\left(\frac{\partial u}{\partial x^{N}}\right)^{2}+q u^{2}\right] d V
$$

We now define the numbers $\lambda_{1}^{(h)} \leqq \lambda_{2}^{(h)} \leqq \cdots$ as the successive minima of a ratio of quadratic forms:

$$
\lambda^{(h)}=\min _{w \in R_{h}} \frac{Q(w)}{h^{N} \sum_{R_{h}} \bar{r} w^{2}} .
$$

The $\lambda_{i}^{(h)}$ are eigenvalues of the finite difference problem

$$
\begin{aligned}
& L^{(h)} w+\bar{q} w=\lambda^{(h)} \bar{r} w, \\
& w \in M_{h},
\end{aligned}
$$

where

$$
\begin{aligned}
& L^{(h)} w\left(m^{i} h\right)=-h^{-2} \sum_{j=1}^{N}\left\{\overline{p^{j}}\left(m^{i} h\right)\left[w\left(m^{i} h+\delta_{i j} h\right)-w\left(m^{i} h\right)\right]\right. \\
& \left.-\overline{p^{j}}\left(m^{i} h-\delta_{i j} h\right)\left[w\left(m^{i} h\right)-w\left(m^{i} h-\delta_{i j} h\right)\right]\right\} .
\end{aligned}
$$

The equation (3.21) is clearly a finite difference analogue of (3.1).

We now proceed exactly as in $\S 2$ to let

$$
u=\xi_{1} u_{1}+\cdots+\xi_{k} u_{k}
$$

where the $u_{i}$ are the normalized eigenfunctions of (3.1). Then

$$
v=\xi_{1} v_{1}+\cdots+\xi_{k} v_{k}
$$

where the $v_{i}$ are related to the $u_{i}$ by (3.4). We apply the Poincare inequality

$$
\lambda_{k}^{(h)} \leqq \max _{\xi_{1}, \cdots, \xi_{k}} \frac{Q\left(\xi_{1} v_{1}+\cdots+\xi_{k} v_{k}\right)}{h^{-N} \sum_{R_{h}} \overline{\bar{r}\left(\xi_{1} v_{1}+\cdots+\xi_{k} v_{k}\right)^{2}}}
$$

together with the inequalities (3.19) and (3.11) to find

$$
\lambda_{k}^{(h)} \leqq \frac{\lambda_{k}}{1-\frac{h^{2}}{\pi^{2} r_{m}}\left[\sqrt{K \lambda_{k}}+\sqrt{L}\right]^{2}} .
$$

Solving for $\lambda_{k}$ we obtain the lower bound 


$$
\lambda_{k} \geqq \lambda_{k}^{(h)}\left[\frac{\left\{1+\frac{h^{2}}{\pi^{2} r_{m}}\left(K \lambda_{k}^{(h)}-L\right)\right\}^{1 / 2}-\frac{h^{2}}{\pi^{2} r_{m}}\left\{K L \lambda_{k}^{(h)}\right\}^{1 / 2}}{1+\frac{h^{2}}{\pi^{2} r_{m}} K \lambda_{k}^{(h)}}\right]^{2} .
$$

Clearly this lower bound differs from $\lambda_{k}^{(h)}$ only by a term of order $h^{2}$. It should be noted that it is independent of $N$ and, except for $\lambda_{k}^{(h)}$ itself, of $k$. For the case of the Laplace operator treated in section $2, K=r_{m}=1$ and $L=0$. Then (3.27) reduces to (2.25).

We note that (3.27) simplifies considerably when the function $r$ is constant so that $L=0$.

4. The general self-adjoint case. In the preceding section we restricted ourselves to the differential equation (3.1), where no mixed derivatives occur. In this section we shall treat the general case

$$
\begin{array}{cc}
-\sum_{i, j=1}^{N} \frac{\partial}{\partial x^{i}}\left(a^{i j} \frac{\partial u}{\partial x^{j}}\right)+q u=\lambda r u & \text { in } R, \\
u=0 & \text { on } \dot{R} .
\end{array}
$$

Here $\mathrm{a}^{i j}$ is assumed to be a uniformly positive definite symmetric matrix in $R, r$ is assumed positive, and $q$ non-negative. All coefficients are taken as piecewise differentiable.

We keep the notation of $\S 3$. In particular, we consider the continuous function $u$ vanishing outside $R$, and the mesh function $v$ in $M_{h}$ defined by (3.4).

The inequalities (3.11) and (3.13) can be used almost without change. The problem is to find a quadratic form in $v$ which can be bounded from above in terms of the quadratic form

$$
\int_{R}\left[\sum_{i, j=1}^{N} a^{i j} \frac{\partial u}{\partial x^{i}} \frac{\partial u}{\partial x^{j}}+q u^{2}\right] d V,
$$

and which approximates this form for small $h$.

We begin with the identity

$$
\begin{aligned}
& \int_{R} \sum_{i, j=1}^{N} a^{i j} \frac{\partial u}{\partial x^{i}} \frac{\partial u}{\partial x^{j}} d V-h^{N} \sum_{R_{h}} \sum_{i, j=1}^{N} \overline{a^{i j}} w_{i} w_{j} \\
= & \sum_{R_{h}} \int_{0 \leqq \alpha^{p} \leqq h} \sum_{i, j, k, l=1}^{N} a_{i j}\left[a^{i k} \frac{\partial u}{\partial x^{k}}\left(m^{p} h+\alpha^{p}\right)-\overline{a^{i k}} w_{k}\right]\left[a^{j l} \frac{\partial u}{\partial x^{l}}\left(m^{p} h+\alpha^{p}\right)\right. \\
& \left.-\bar{a}^{j l} w_{l}\right] d \alpha^{1} \cdots d \alpha^{N} \geqq 0 .
\end{aligned}
$$

Here $a_{i j}$ is the inverse matrix of $a^{i j}$, and we have defined the mesh matrix $^{3}$ 


$$
\overline{a^{i j}}\left(m^{l} h\right) \equiv\left[h^{-N} \int_{0 \leqq \alpha^{l} \leqq h} a_{i j}\left(m^{l} h+\alpha^{l}\right) d \alpha^{1} \cdots d \alpha^{N}\right]^{-1},
$$

i.e.,

$$
\sum_{p=1}^{N}\left[h^{-N} \int_{0 \leqq \alpha^{l} \leqq h} a_{i p}\left(m^{l} h+\alpha^{l}\right) d \alpha^{1} \cdots d \alpha^{N}\right] \bar{\alpha}^{p j}\left(m^{l} h\right)=\delta_{i j},
$$

and the mesh vector

$$
w_{k}\left(m^{l} h\right) \equiv\left[h^{-N} \int_{0 \leqq \alpha^{l} \leqq h} \frac{\partial u}{\partial x^{k}}\left(m^{l} h+\alpha^{l}\right) d \alpha^{1} \cdots d \alpha^{N}\right] .
$$

While $w_{k}$ is clearly an approximation to $\partial u / \partial x^{k}$, it cannot be obtained from $v$ or any other mesh function. Therefore, (4.3) does not give a quadratic form in $v$. However, since the finite difference

$$
d_{k}[v]\left(m^{i} h\right) \equiv h^{-1}\left[v\left(m^{i} h+\delta_{i k} h\right)-v\left(m^{i} h\right)\right]
$$

also approximates $\partial u / \partial x^{k}$, it must approximate $w_{k}$. We estimate the error introduced by using $d_{k}[v]$ instead of $w_{k}$. It follows from the triangle inequality that

$$
\begin{aligned}
& \left\{h^{N} \sum_{R_{h}}\left[\sum_{i, j=1}^{N} \overline{a^{i j}} d_{i}[v] d_{j}[v]+\bar{q} v^{2}\right]\right\}^{1 / 2} \leqq\left\{h^{N} \sum_{R_{h}}\left[\sum_{i, j} \overline{a^{i j}} w_{i} w_{j}+\bar{q} v^{2}\right]\right\}^{1 / 2} \\
& \quad+\left\{h^{N} \sum_{R_{h}} \sum_{i, j} \overline{a^{i j}}\left(w_{i}-d_{i}[v]\right)\left(w_{j}-d_{j}[v]\right)\right\}^{1 / 2} .
\end{aligned}
$$

It can be seen from the definition (4.4) that largest and smallest eigenvalues of $\overline{a^{i j}}$ lie between the maximum of the largest eigenvalue and minimum of the smallest eigenvalue of $a^{i j}$ in the cube of definition. Hence, $\bar{a}^{i j}$ is still positive definite so that the triangle inequality applies. The first term on the right of (4.8) is bounded by means of (4.3). The second term is the error due to replacing $w_{k}$ by $d_{k}[v]$. We shall bound it.

Let the constant $a$ be a uniform upper bound for the eigenvalues of $a^{i j}$; that is,

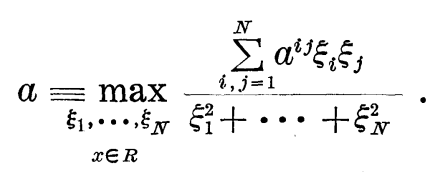

Then the same bound holds if $a^{i j}$ is replaced by $\bar{a}^{i j}$. Hence,

$$
h^{N} \sum_{R_{h}} \sum_{i, j=1}^{N} \overline{a^{i j}}\left(w_{i}-d_{i}[v]\right)\left(w_{j}-d_{j}[v]\right) \leqq a h^{N} \sum_{R_{h}}^{N} \sum_{i=1}\left(w_{i}-d_{i}[v]\right)^{2} .
$$

We use the identity 


$$
\begin{aligned}
w_{i}\left(m^{\imath} h\right)-d_{i}[v]\left(m^{l} h\right) & =\int_{\substack{0 \leqq \alpha^{i} \leqq 2 h \\
0 \leqq \alpha^{\leqq} \leqq h, l \neq i}} \varphi\left(\alpha^{i}\right) \frac{\partial^{2} u}{\partial x^{i 2}}\left(m^{l} h+\alpha^{l}\right) d \alpha^{1} \cdots d \alpha^{N} \\
& -\oint \varphi\left(\alpha^{i}\right) \frac{\partial u}{\partial x^{i}} \frac{\partial \alpha^{i}}{\partial n} d S_{\alpha}
\end{aligned}
$$

where

$$
\varphi(\alpha)= \begin{cases}\frac{1}{2} h^{-N-1} \alpha(2 h-\alpha) & 0 \leqq \alpha \leqq h, \\ \frac{1}{2} h^{-N-1}(2 h-\alpha)^{2} & h \leqq \alpha \leqq 2 h .\end{cases}
$$

The volume integral is actually over the intersection of the rectangular parallelepiped with $R$. On the boundaries of the parallelepiped the integrand of the surface integral vanishes by the construction of $\varphi$. Thus, the last integal is only over the part of $\dot{R}$ cut by the parallelepiped.

We apply Schwarz's inequality and the triangle inequality to (4.11), and note that $R$ is covered twice by each set of parallelepipeds. Using the fact that $u=0$ on $\dot{R}$ we have

$$
\begin{gathered}
\left\{h^{N} \sum_{R_{h}} \sum_{i=1}^{N}\left(w_{i}-d_{i}[v]\right)^{2}\right\}^{1 / 2} \leqq\left\{\frac{11}{30} h^{2} \sum_{i=1}^{N} \int_{R}\left(\frac{\partial^{2} u}{\partial x^{i 2}}\right)^{2} d V\right\}^{1 / 2} \\
+\left\{h^{N} \sum_{R_{h}} \sum_{i=1}^{N}\left[\oint F\left(\frac{\partial u}{\partial n}\right)^{2} d S_{\alpha} \oint \frac{\varphi^{2}}{F}\left(\frac{\partial \alpha^{i}}{\partial n}\right)^{4} d S_{\alpha}\right\}^{1 / 2} .\right.
\end{gathered}
$$

Here $F$ is an arbitrary positive function defined on $\dot{R}$. To estimate the last term on the right, we note that $\oint\left|\partial \alpha^{i} / \partial n\right| d S_{\alpha}$ represents the projection perpendicular to the $x^{i}$-axis of the total surface. We call $\nu_{h}$ the maximum number of intersections of $\dot{R}$ with any line segment of length $2 h$ parallel to one of the coordinate axes. Clearly, $\nu_{h}$ is a monotone increasing function of $h$. If $\dot{R}$ is at all regular, $\nu_{h}$ is bounded, and equals 2 for sufficiently small $h$. Noting that $\varphi^{2} \leqq \frac{1}{4} h^{2-2}{ }_{N},\left|\partial \alpha^{i} / \partial n\right| \leqq 1$, and that the projection of any one layer of area within the parallelepiped in the $x^{i}$-direction is at most $h^{N-1}$ we have

$$
\oint \frac{\varphi^{2}}{F}\left(\frac{\partial \alpha^{i}}{\partial n}\right)^{4} d S_{\alpha} \leqq \frac{\nu_{h} h^{1-N}}{4 F_{m}},
$$

where

$$
F_{m}=\min _{x \in \dot{R}} F,
$$


Again taking account of the fact that $R$ is covered twice by each set of parallelepipeds, we have

$$
\begin{aligned}
& \left\{h^{N} \sum_{R_{h}} \sum_{i=1}^{N}\left(w_{i}-d_{i}[v]^{2}\right)\right\}^{1 / 2} \leqq\left\{\frac{11}{30} h^{2} \sum_{i=1}^{N} \int_{R}\left(\frac{\partial^{2} u}{\partial x^{i 2}}\right)^{2} d V\right\}^{1 / 2} \\
& \quad+\left\{\frac{N \nu_{h} h}{2 F_{m}} \oint_{\dot{R}} F\left(\frac{\partial u}{\partial n}\right)^{2} d S\right\}^{1 / 2} .
\end{aligned}
$$

It thus becomes necessary to bound the integral of the sum of squares of the second derivatives of $u$, and a boundary integral of the square of the normal derivative of $u$. We begin with the latter.

We utilize an identity which was found for the Laplace operator by F. Rellich [16], for hyperbolic operators by L. Hörmander [9], and which was extensively used for purposes similar to the present one by L. E. Payne and the author $[11,12,13]$. Let $f^{1}(x), \cdots, f_{N}(x)$ be an arbitrary piecewise differentiable vector field in $R$. The identity is

$$
\begin{aligned}
& \oint\left(\sum_{k=1}^{N} f^{k} n_{k}\right)\left(\sum_{i, j=1}^{N} a^{i j} n_{i} n_{j}\right)\left(\frac{\partial u}{\partial n}\right)^{2} d S \\
= & \int_{R i, j, k=1} \sum^{N}\left(\frac{\partial f^{k}}{\partial x^{k}} a^{i j}-2 \frac{\partial f^{i}}{\partial x^{k}} a^{j k}+f^{k} \frac{\partial a^{i j}}{\partial x^{k}}\right) \frac{\partial u}{\partial x^{i}} \frac{\partial u}{\partial x^{j}} d V \\
& -\int_{R} 2 \mathscr{A}(u) \sum_{i=1}^{N} f^{i} \frac{\partial u}{\partial x^{i}} d V,
\end{aligned}
$$

where we have written

$$
\mathscr{A}(u) \equiv \sum_{i, j=1}^{N} \frac{\partial}{\partial x^{i}}\left(a^{i j} \frac{\partial u}{\partial x^{j}}\right),
$$

and $n_{k}$ is the outward unit normal on $\dot{R}$.

We now assume that the vector field $f^{k}$ has the property that its outward normal component on $\dot{R}$ is positive :

$$
\sum_{k=1}^{N} f^{k} n_{k}>0
$$

Then we can put

$$
F \equiv \sum_{k=1}^{N} f^{k} n_{k} \sum_{i, j=1}^{N} a^{i j} n_{i} n_{j}
$$

in (4.16). For example, if $R$ is star-shaped with respect to the origin, we may take $f^{k}=x^{k}$. More generally, if $\dot{R}$ is represented by an equation $R(x)=0$ where $R(x)$ is a twice differentiable function in $R$ whose outward normal derivative on $\dot{R}$ is positive, we may take $f^{k}=\partial R / \partial x^{k}$. It still 
remains to bound the right hand side of (4.17). For this purpose, we restrict ourselves to the function

$$
u=\xi_{1} u_{1}+\cdots+\xi_{k} u_{k}
$$

where $u_{1}, \cdots, u_{k}$ are the first $k$ eigenfunctions of (4.1) normalized by (3.3). Then

$$
\mathscr{A}(u)=\sum_{n=1}^{k} \xi_{n}\left(q-\lambda_{n} r\right) u
$$

The integrand of the first integral on the right of (4.17) is a quadratic form in the gradient of $u$. Since the lowest eigenvalue of $a^{i j}$ is assumed to be positive and bounded away from zero, there exists a constant $c$ defined by

$$
c \equiv \max _{\eta_{1}, \ldots, \eta_{N}} \frac{\sum_{i, j, k=1}^{N}\left(\frac{\partial f^{k}}{\partial x^{k}} r^{i j}-2 \frac{\partial f^{i}}{\partial x^{k}} a^{j k}+f^{k} \frac{\partial a^{i j}}{\partial x^{k}}\right) \eta_{i} \eta_{j}}{\sum_{i, j=1}^{N} a^{i j} \eta_{i} \eta_{j}} .
$$

Thus, the first integral on the right of (4.17) is bounded by

$$
\begin{aligned}
c \int_{R} \sum_{i, j=1}^{N} a^{i j} \frac{\partial u}{\partial x^{i}} \frac{\partial u}{\partial x^{j}} d V & \leqq c \int_{R}\left[\sum_{i, j=1}^{N} a^{i j} \frac{\partial u}{\partial x^{i}} \frac{\partial u}{\partial x^{j}}+q u^{2}\right] d V \\
& =c\left(\lambda_{1} \xi_{1}^{2}+\cdots+\lambda_{k} \xi_{k}^{2}\right) .
\end{aligned}
$$

Substituting (4.21) in the second integral and using Schwarz's inequality, we find the bound

$$
\begin{aligned}
& M_{1}\left\{\lambda_{1} \xi_{1}^{2}+\cdots+\lambda_{k} \xi_{k}^{2}\right\}^{1 / 2}\left\{\lambda_{1}^{2} \xi_{1}^{2}+\cdots+\lambda_{k}^{2} \xi_{k}^{2}\right\}^{1 / 2} \\
& \quad+M_{2}\left\{\lambda_{1} \xi_{1}^{2}+\cdots+\lambda_{k} \xi_{k}^{2}\right\}^{1 / 2}\left\{\xi_{1}^{2}+\cdots+\xi_{k}^{2}\right\}^{1 / 2},
\end{aligned}
$$

where

$$
M_{1}=2 \max \left\{r \sum_{i, j=1}^{N} a_{i j} f^{i} f^{j}\right\}^{1 / 2}, \quad M_{2}=2 \max \left\{\frac{q^{2}}{r} \sum_{i, j=1}^{N} a_{i j} f^{i} f^{j}\right\}^{1 / 2} .
$$

Thus we find

$$
\begin{aligned}
& \oint_{\dot{R}} F\left(\frac{\partial u}{\partial n}\right)^{2} d S \leqq c\left(\lambda_{1} \xi_{1}^{2}+\cdots+\lambda_{k} \xi_{k}^{2}\right) \\
& \quad+M_{1}\left\{\lambda_{1} \xi_{1}^{2}+\cdots+\lambda_{k} \xi_{k}^{2}\right\}^{1 / 2}\left\{\lambda_{1}^{2} \xi_{1}^{2}+\cdots+\lambda_{k}^{2} \xi_{k}^{2}\right\}^{1 / 2} \\
& \quad+M_{2}\left\{\lambda_{1} \xi_{1}^{2}+\cdots+\lambda_{k} \xi_{k}^{2}\right\}^{1 / 2}\left\{\xi_{1}^{2}+\cdots+\xi_{k}^{2}\right\}^{1 / 2} .
\end{aligned}
$$

We now estimate the first integral on the right of (4.16). For this purpose we extend an argument used in the case of the twodimensional Laplace operator by L. E. Payne [10]. 
We let

$$
a^{i j}=\sqrt{g} g^{i j}
$$

where

$$
\begin{aligned}
& g=\operatorname{det}\left[g_{i j}\right], \\
& g_{i j}=\left[g^{i j}\right]^{-1} .
\end{aligned}
$$

In three or more dimensions one can solve (4.27):

$$
\begin{aligned}
g & =\left\{\operatorname{det}\left[a^{i j}\right]\right\}^{-(2 / N-2)}, \\
g^{k l} & =\left\{\operatorname{det}\left[a^{i j}\right]\right\}^{1 / N-2} a^{k l} .
\end{aligned}
$$

In two dimensions (4.27) implies $\operatorname{det}\left[a^{i j}\right]=1$. If this is satisfied, one takes $g^{i j}=a^{i j}$. If not, one must make a change of dependent and independent variables to arrive at $\operatorname{det}\left[a^{i j}\right]=1$. We assume this to have been done.

We consider $g_{i j}$ as the metric tensor of a Riemannian space. We derive the tensor identity (using summation convention)

$$
\begin{aligned}
\sqrt{g} g^{k l}\left(g^{i j} u_{1 i} u_{1 j}\right)_{1 k l} & =2 \sqrt{g} g^{k l} g^{i j}\left[u_{1 \cdot k} u_{1 j l}+u_{1 i} u_{1 j k l}\right] \\
& =2 \sqrt{g} g^{k l} g^{i j}\left[u_{1 i k} u_{1 j l}+u_{1 i} u_{1 k l j}+u_{1 i} R_{k j l}^{p} u_{1 p p}\right] \\
& =2 \sqrt{g} g^{k l} g^{i j} u_{1 i k} u_{1 j l}+2 \sqrt{g} g^{i j} u_{1}\left(g^{k l} u_{1 k l}\right)_{1 j} \\
& -2 \sqrt{g} R^{i j} u_{1 i} u_{1 j} .
\end{aligned}
$$

Here we have used symbol ${ }_{1 i}$ for covariant differentiation. $R_{k j l}^{p}$ is the Riemann curvature tensor, and $R^{i j}$ is the contravariant Ricci tensor (see, for example, [17]) :

$$
\begin{aligned}
R^{i s} & \equiv g^{i l} g^{j m}\left[\frac{1}{2} \frac{\partial^{2}}{\partial x^{l} \partial x^{m}} \ln g\right. \\
& \left.-\frac{1}{2}\left\{\begin{array}{c}
p \\
l m
\end{array}\right\} \frac{\partial}{\partial x^{p}} \ln g-\frac{\partial}{\partial x^{p}}\left\{\begin{array}{c}
p \\
l m
\end{array}\right\}+\left\{{ }_{l} p_{q}\right\}\left\{\begin{array}{c}
q \\
n p
\end{array}\right\}\right],
\end{aligned}
$$

where

$$
\left\{\begin{array}{c}
p \\
l m
\end{array}\right\} \equiv \frac{1}{2} g^{p q}\left[\frac{\partial g^{q l}}{\partial x^{m}}+\frac{\partial g^{q m}}{\partial x^{l}}-\frac{\partial g^{l m}}{\partial x^{q}}\right]
$$

is the Christoffel symbol of the second kind. We have

$$
\begin{gathered}
u_{1 i} \equiv \frac{\partial u}{\partial x^{i}}, \\
u_{1 i j}=u_{1 j i} \equiv \frac{\partial^{2} u}{\partial x^{i} \partial x^{1}}-\left\{\begin{array}{c}
p \\
i \quad j
\end{array}\right\} \frac{\partial u}{\partial x^{p}},
\end{gathered}
$$

and consequently 


$$
g^{k l} u_{\mid k l}=\mathscr{A}(u) / \sqrt{g}
$$

where $\mathscr{A}(u)$ is the operator (4.18). The left-hand side of (4.30) is a perfect divergence. Integrating (4.30) over $R$, applying the divergence theorem, and transposing terms gives, after use of (4.34),

$$
\begin{aligned}
& \int_{R} \sqrt{g} g^{k l} g^{i j} u_{\mid i k} u_{\mid j l} d V=\frac{1}{2} \oint_{\dot{R}} a^{k l} \frac{\partial}{\partial x^{k}}\left(g^{i j} \frac{\partial u}{\partial x^{i}} \frac{\partial u}{\partial x^{j}}\right) n_{l} d S \\
& -\int_{R} a^{i j} \frac{\partial u}{\partial x^{i}} \frac{\partial}{\partial x^{j}}\left(\frac{\mathscr{A}(u)}{\sqrt{g}}\right) d V+\int_{R} \sqrt{g} R^{i j} \frac{\partial u}{\partial x^{i}} \frac{\partial u}{\partial x^{j}} d V .
\end{aligned}
$$

Here we have used Euclidean elements of volume and area.

We now restrict ourselves to functions $u$ of the form (4.20), so that $\mathscr{A}(u)=0$ on the boundary. Then by the divergence theorem and (4.27)

$$
-\int_{R} a^{i j} \frac{\partial u}{\partial x^{i}} \frac{\partial}{\partial x^{j}}\left(\frac{\mathscr{A}(u)}{\sqrt{g}}\right) d V=\int_{R} \frac{1}{\sqrt{g}} \mathscr{A}(u)^{2} d V .
$$

But when $u$ is given by (4.20), $\mathscr{A}(u)$ is given by (4.21). By the triangle inequality we find the bound

(4.37) $\left\{\int_{R} \frac{1}{\sqrt{g}} \mathscr{A}(u)^{2} d V\right\}^{1 / 2} \leqq\left\{l_{1}\left(\xi_{1}^{2}+\cdots+\xi_{k}^{2}\right)\right\}^{1 / 2}+\left\{l_{2}\left(\lambda_{1}^{2} \xi_{1}^{2}+\cdots+\lambda_{k}^{2} \xi_{k}^{2}\right)\right\}^{1 / 2}$

with

$$
l_{1}=\max \left(\frac{q^{2}}{r v g}\right)
$$

and

$$
l_{2}=\max \left(\frac{r}{\sqrt{g}}\right) .
$$

For the last term on the right of (4.35) we put

$$
d \equiv \max _{\substack{\eta_{1}, \cdots, \eta_{N} \\ x \in R}}\left(\frac{\sqrt{g} R^{i j} \eta_{i} \eta_{j}}{a^{i j} \eta_{i} \eta_{j}}\right) .
$$

Then

(4.41) $\int_{R} \sqrt{g} R^{i j} \frac{\partial u}{\partial x^{i}} \frac{\partial u}{\partial x^{j}} d V \leqq d \int_{R} a^{i j} \frac{\partial u}{\partial x^{i}} \frac{\partial u}{\partial x^{j}} d V \leqq d\left(\lambda_{1} \xi_{1}^{2}+\cdots+\lambda_{k} \xi_{k}^{2}\right)$.

We come now to the surface integral in (4.35). We suppose that in some neighborhood of the surface $\dot{R}$ there is defined a differentiable function $R(x)$ vanishing on $\dot{R}$ and such that the outward normal derivative is positive. Since $R(x)$ vanishes on $\dot{R}$ we may put 


$$
u(x)=R(x) \varphi(x)
$$

in the neighborhood of $\dot{R}$. Then we see that on $\dot{R}$

$$
\begin{aligned}
& a^{k l} \frac{\partial}{\partial x^{k}}\left(g^{i j} \frac{\partial u}{\partial x^{i}} \frac{\partial u}{\partial x^{j}}\right) n_{l} \\
= & \left(\frac{\partial R}{\partial n}\right)^{-1}\left[4 \varphi g^{k l} \frac{\partial R}{\partial x^{k}} \frac{\partial R}{\partial x^{l}} a^{i j} \frac{\partial R}{\partial x^{i}} \frac{\partial \varphi}{\partial x^{j}}+\varphi^{2} a^{i j} \frac{\partial R}{\partial x^{i}} \frac{\partial}{\partial x^{j}}\left(g^{k l} \frac{\partial R}{\partial x^{k}} \frac{\partial R}{\partial x^{l}}\right)\right] .
\end{aligned}
$$

Also on $\dot{R}$

$$
\mathscr{A}(u)=\varphi \mathscr{A}(R)+2 a^{i j} \frac{\partial R}{\partial x^{i}} \frac{\partial \varphi}{\partial x^{j}} .
$$

Since $u$ is taken of the form (4.20) and the $u_{i}$ satisfy (4.1), $\mathscr{A}(u)$ vanishes on $\dot{R}$. Hence, we may eliminate the derivaties of $\varphi$ occurring in (4.43) by setting (4.44) equal to zero. Finally, to identify $\varphi$ in terms of $u$ we take the normal derivative of (4.42) to find

$$
\frac{\partial u}{\partial n}=\varphi \frac{\partial R}{\partial n} \text {. }
$$

Thus, we arrive at

$$
\begin{aligned}
& a^{k l} \frac{\partial}{\partial x^{k}}\left(g^{i j} \frac{\partial u}{\partial x^{i}} \frac{\partial u}{\partial x^{j}}\right) n_{l} \\
= & -2\left[g^{p q} n_{p} n_{q}\right]^{3 / 2} \frac{\partial}{\partial x^{i}}\left(\left\{g^{k l} \frac{\partial R}{\partial x^{k}} \frac{\partial R}{\partial x^{l}}\right\}^{-1 / 2} \sqrt{g} g^{i j} \frac{\partial R}{\partial x^{j}}\right)\left(\frac{\partial u}{\partial n}\right)^{2} .
\end{aligned}
$$

The coefficient of $(\partial u / \partial n)^{2}$ is clearly independent of the particular function $R(x)$ used to represent $\dot{R}$. It is a local geometric property of $\dot{R}$. In fact, if $g^{i j}$ is the unit matrix, the coefficient is just $-2(N-1)$ times the mean curvature of $\dot{R}$, as can be seen by taking for $R(x)$ the distance from $\dot{R}$. If $g_{i s}$ is the metric of a flat space, the divergence term is still proportional to the mean curvature of $\dot{R}$ in this space. The first part of the coefficient arises from the fact that we are mixing a Euclidean and a non-Euclidean metric.

Setting

$$
\text { (4.47) } e \equiv \max \left\{-F^{-1}\left[g^{p q} n_{p} n_{q}\right]^{3 / 2} \frac{\partial}{\partial x^{i}}\left(\left\{g^{k l} \frac{\partial R}{\partial x^{k}} \frac{\partial R}{\partial x^{l}}\right\}^{-1 / 2} \sqrt{g} g^{i j} \frac{\partial R}{\partial x^{j}}\right)\right\}
$$

where $F$ is defined by (4.19) in terms of the arbitrary vector field pointing outward on $\dot{R}$, we have by $(4.26)$ 


$$
\begin{aligned}
& \frac{1}{2} \oint_{\dot{R}} a^{k l} \frac{\partial}{\partial x^{k}}\left(g^{i j} \frac{\partial u}{\partial x^{i}} \frac{\partial u}{\partial x^{j}}\right) n_{l} d S \leqq e \oint F\left(\frac{\partial u}{\partial n}\right)^{2} d S \\
\leqq & c e\left(\lambda_{1} \xi_{1}^{2}+\cdots+\lambda_{k} \xi_{k}^{2}\right)+M_{1} e\left\{\lambda_{1} \xi_{1}^{2}+\cdots+\lambda_{k} \xi_{k}^{2}\right\}^{1 / 2}\left\{\lambda_{1}^{2} \xi_{1}^{2}+\cdots+\lambda_{k}^{2} \xi_{k}^{2}\right\}^{1 / 2} \\
& +M_{2} e\left\{\lambda_{1} \xi_{1}^{2}+\cdots+\lambda_{k} \xi_{k}^{2}\right\}^{1 / 2}\left\{\xi_{1}^{2}+\cdots+\xi_{k}^{2}\right\}^{1 / 2} .
\end{aligned}
$$

We note that in order to have a finite $e$ it is necessary to assume that the coefficient of $(\partial u / \partial n)^{2}$ in $(4.46)$ is bounded above. Since this coefficient, at least in a flat space, is proportional to the negative of the mean curvature, one sees that this implies that $\dot{R}$ has no re-entrant corners, edges, or cusps. On the other hand, non-re-entrant corners, edges, and cusps cause no difficulty. It is easily ascertained from the asymptotic form of a solution of (4.1) that the integrals of the squares of the second derivatives, which we are seeking to bound, actually diverge at re-entrant corners, edges, and cusps.

Having bounded the right-hand side of (4.35), we turn to the lefthand side. The positive definite symmetric matrix $g^{i j}$ may be expanded in terms of its eigenvalues $0<\mu_{1} \leqq \mu_{2} \leqq \cdots \leqq \mu_{N}$ and orthonormal eigenvectors in the form

$$
g^{i j}=\sum_{p=1}^{N} \mu_{p} c_{p}^{i} c_{p}^{j}
$$

Then

$$
\begin{aligned}
g^{k l} g^{i j} u_{1 i k} u_{1 j l} & =\sum_{p, q, i, k, j, l=1}^{N} \mu_{p} \mu_{q}\left(c_{p}^{i} c_{q}^{k} u_{1 i k}\right)\left(c_{p}^{j} c_{q}^{l} u_{1 j l}\right) \\
& \geqq \mu_{1}^{2} \sum_{p, q=1}^{N}\left(\sum_{i, k=1}^{N} c_{p}^{i} c_{q}^{k} u_{l i k}\right)^{2} \\
& =\mu_{1}^{2} \sum_{i, k=1}^{N} u_{1 i k}^{2},
\end{aligned}
$$

the last equality being due to the orthonormality of the eigenvectors. Now by virtue of (4.33) and the triangle inequality

$$
\begin{aligned}
\left\{\int_{R} \sum_{i=1}^{N}\left(\frac{\partial^{2} u}{\partial x^{i 2}}\right)^{2} d V\right\}^{1 / 2} \leqq & \left\{\int_{R} \sum_{i, k=1}^{N} u_{1 i k}^{2} d V\right\}^{1 / 2} \\
& \left.+\left\{\int_{R} \sum_{i=1}^{N}\left[\sum_{p=1}^{N}\left\{\begin{array}{c}
p \\
i
\end{array}\right\}\right) \frac{\partial u}{\partial x^{p}}\right]^{2} d V\right\}^{1 / 2} .
\end{aligned}
$$

Thus, letting

$$
b \equiv \max \left(\frac{1}{\mu_{1}^{2} \sqrt{g}}\right)=\max _{\substack{\eta_{1}, \cdots, \eta_{N} \\ x \in R}}\left(\frac{\eta_{1}^{2}+\cdots+\eta_{N}^{2}}{\sum_{i, j=1}^{N} a^{i j} \eta_{i} \eta_{j}}\right)^{2} \sqrt{g}
$$




$$
m=\max \sum_{i, p, q=1}^{N} a_{p q}\left\{\begin{array}{c}
p \\
i
\end{array}\right\}\left\{\begin{array}{c}
q \\
i
\end{array} i\right\}
$$

and applying Schwarz's inequality we have

$$
\begin{aligned}
\left\{\int_{R} \sum_{i=1}^{N}\left(\frac{\partial^{2} u}{\partial x^{i 2}}\right)^{2} d V\right\}^{1 / 2} & \leqq\left\{\int_{R} \sqrt{g} g^{k l} g^{i j} u_{1 i k} u_{1 j l} d V\right\}^{1 / 2} \\
& +\left\{m \int_{R} a^{p q} \frac{\partial u}{\partial x^{p}} \frac{\partial u}{\partial x^{q}} d V\right\}^{1 / 2}
\end{aligned}
$$

We return now to the original problem. We define the quadratic functional $Q(w)$ of mesh function $w$ by

$$
Q(w) \equiv h^{N} \sum_{R_{h}}\left\{\sum_{i, j=1}^{N} \bar{a}^{i j} d_{i}[w] d_{j}[w]+g w^{2}\right\}
$$

where $d_{i}$ is the first difference operator in the $x^{i}$ direction defined by (4.7), and $\bar{a}^{i j}$ and $\bar{q}$ are the average functions defined by (4.9) and (3.14).

We let $\lambda_{1}^{(h)} \leqq \lambda_{2}^{(h)} \leqq \cdots$ be the successive minima of the ratio

$$
\frac{Q(w)}{h^{N} \sum_{R_{h}} \bar{r} w^{2}}
$$

with respect to mesh functions $w$ in $M_{h}$. Here $\bar{r}$ is defined by (3.5). The minimizing functions and the minima satisfy the finite difference equation

$$
\text { (4.57) }-\sum_{i, j=1}^{N} d_{i}\left[\bar{a}^{i j} d_{j}[w]\right]\left(m^{\imath} h-\delta_{i l} h\right)+\bar{q}\left(m^{\imath} h\right) w\left(m^{\imath} h\right)=\lambda^{(h)} \bar{r}\left(m^{\imath} h\right) w\left(m^{\imath} h\right) .
$$

This is, of course, a finite difference analogue of (4.1).

The Poincaré inequality (3.25) still holds. Taking for $v$ the mesh function defined by (3.4) and for $u$ the linear combination $\xi_{1} u_{1}+\cdots+\xi_{k} u_{k}$ of the first $k$ eigenfunctions of (4.1), we get $v$ in the form $\xi_{1} v_{1}+\cdots+\xi_{k} v_{k}$.

We now put together the inequalities (4.8), (3.13), (4.3), (4.10), (4.16), (4.54), (4.35), (4.48), (4.37), (4.41), and (4.26) to find

$$
\begin{aligned}
& \left\{Q\left(\xi_{1} v_{1}+\cdots+\xi_{k} v_{k}\right)\right\}^{1 / 2} \leqq\left\{\sum_{i=1}^{k} \lambda_{i} \xi_{i}^{2}\right\}^{1 / 2} \\
& +h\left\{\frac{11}{30} a b\right\}^{1 / 2}\left\{(c e+d) \sum \lambda_{i} \xi_{i}^{2}+M_{1} e\left[\sum \lambda_{i} \xi_{i}^{2} \sum \lambda_{j}^{2} \xi_{j}^{2}\right]^{1 / 2}\right. \\
& \left.+M_{2} e\left[\sum \lambda_{i} \xi_{i}^{2} \sum \xi_{j}^{2}\right]^{1 / 2}+\left[\left(l_{1} \sum \xi_{i}^{2}\right)^{1 / 2}+\left(l_{2} \sum \lambda_{i}^{2} \xi_{i}^{2}\right)^{1 / 2}\right]^{2}\right\}^{1 / 2} \\
& +h\left\{\frac{11}{30} a m \sum \lambda_{i} \xi_{i}^{2}\right\}^{1 / 2}+h^{1 / 2}\left\{\frac{N_{\nu_{h}} a}{2 F_{m}}\right\}^{1 / 2} \\
& \quad \times\left\{c \sum \lambda_{i} \xi_{i}^{2}+M_{1}\left[\sum \lambda_{i} \xi_{i}^{2} \sum \lambda_{j}^{2} \xi_{j}^{2}\right]^{1 / 2}+M_{2}\left[\sum \lambda_{i} \xi_{i}^{2} \sum \xi_{j}^{2}\right]^{1 / 2}\right\}^{1 / 2}
\end{aligned}
$$


The denominator is bounded by the generalization of (3.11), namely

$$
h^{N} \sum_{R_{h}} \bar{r} v^{2} \geqq 1-\frac{h^{2}}{\pi^{2} r_{m}}\left[\left\{K \sum_{i=1}^{k} \lambda_{i} \xi_{i}^{2}\right\}^{1 / 2}+\left\{L \sum \xi_{i}^{2}\right\}^{1 / 2}\right]^{2}
$$

where

$$
\begin{aligned}
& K=\max _{\substack{\eta_{1}, \cdots, \eta_{N} \\
x \in R}} \frac{r^{2}\left(\eta_{1}^{2}+\cdots+\eta_{N}^{2}\right)}{\sum_{i, j=1}^{N} a^{i j} \eta_{i} \eta_{j}}, \\
& L=\max \left(\mid \operatorname{grad} r^{2} / \nu\right)
\end{aligned}
$$

Inserting these bounds in the Poincare inequality (3.25) yields

$$
\begin{aligned}
\lambda_{k}^{(h)} \leqq\left[\lambda_{k}^{1 / 2}\right. & +h^{1 / 2}\left\{M_{2} \lambda_{k}^{1 / 2}+c \lambda_{k}+M_{1} \lambda_{k}^{1 / 2}\right\}^{1 / 2}\left\{\frac{N_{\nu_{h}} a}{2 F_{m}}\right\}^{1 / 2}+h\left\{l_{1}+M_{2} e \lambda_{k}^{1 / 2}\right. \\
& \left.+\left(c e+d+2 \sqrt{l_{1} l_{2}}\right) \lambda_{k}+M_{1} \lambda_{k}^{3 / 2}+l_{2} \lambda_{k}^{2}\right\}^{1 / 2}\left\{\frac{11}{30} a b\right\}^{1 / 2} \\
& \left.+h\left\{\frac{11}{30} a m \lambda_{k}\right\}^{1 / 2}\right]^{2}\left[1-\frac{h^{2}}{\pi^{2} r_{m}}\left(\sqrt{K \lambda_{k}}+\sqrt{L}\right)^{2}\right]^{-1} .
\end{aligned}
$$

This is an implicit lower bound for $\lambda_{k}$. We note that the lower bound differs from $\lambda_{k}^{(h)}$ by a term of order $h^{1 / 2}$, rather than $h^{2}$ as in the absence of mixed derivatives. The inequality (4.61) does not reduce to (3.27) when $a^{i j}$ is diagonal.

5. Systems with no mixed derivatives. The process used in $\S 3$ is easily extended to a self-adjoint system of elliptic equations. We must only consider the unknown function in (3.1) as a vector and the coefficients as symmetric matrices. Thus we have

$$
\sum_{\beta=1}^{n}\left\{-\sum_{i=1}^{N} \frac{\partial}{\partial x^{i}}\left(p_{\alpha \beta}^{(i)} \frac{\partial u^{\beta}}{\partial x^{i}}\right)+q_{\alpha \beta} u^{\beta}\right\}=\lambda \sum_{\beta=1}^{n} r_{\alpha \beta} u^{\beta}, \quad \alpha=1, \cdots, n
$$

We assume the matrices $p_{\alpha \beta}^{(1)} \cdots p_{\alpha \beta}^{(N)}$ and $r_{\alpha \beta}$ to be positive definite and $q_{\alpha \beta}$ semi-definite, and all their components piecewise differentiable. We put

$$
v^{\beta}\left(m^{i} h\right) \equiv h^{-N} \int_{0 \leqq \alpha^{i} \leqq h} u^{\beta}\left(m^{i} h+\alpha^{i}\right) d \alpha^{1} \cdots d \alpha^{N}
$$

and, writing $r^{\alpha \beta}$ for the inverse of $r_{\alpha \beta},{ }^{3}$

$$
\bar{r}_{\alpha \beta}\left(m^{i} h\right) \equiv\left[h^{-N} \int_{0 \leqq \alpha i \leqq h} r^{\alpha \beta}\left(m^{i} h+\alpha^{i}\right) d \alpha^{1} \cdots d \alpha^{N}\right]^{-1} .
$$

Then we have, analogous to (3.6) 


$$
\begin{aligned}
& \int_{R} \sum_{\alpha, \beta=1}^{N} r_{\alpha \beta} u^{\alpha} u^{\beta} d V-h^{N} \sum_{R_{h}} \sum_{a, \beta=1}^{n} \bar{r}_{\alpha \beta} v^{\alpha} v^{\beta} \\
& =\sum_{R_{h}} \int_{0 \leqq} \sum_{\alpha} \sum_{\alpha, \beta, \gamma, \delta=1}^{n} r_{\alpha \beta}\left[r_{\alpha \gamma} u^{\gamma}-\bar{r}_{\alpha \gamma} v^{\gamma}\right]\left[r_{h \delta} u^{\delta}-\bar{r}_{\beta \delta} v^{\delta}\right] d \alpha^{1} \cdots d \alpha^{N} .
\end{aligned}
$$

Thus, putting

$$
\begin{aligned}
& r_{m}=\min _{\eta^{1}, \ldots, \eta^{n}}\left[\frac{\sum_{x \in R}^{n} r_{\alpha \beta} \eta^{\alpha} \eta^{\beta}}{\left(\eta^{1}\right)^{2}+\cdots+\left(\eta^{n}\right)^{2}}\right]=\min _{\xi^{1}, \ldots, \xi^{n}}\left[\frac{\xi_{1}^{2}+\cdots+\xi_{n}^{2}}{\sum_{x \in R}^{n} r^{\alpha \beta} \xi_{\alpha} \xi_{\beta}}\right], \\
& K=\max _{\substack{i=1, \cdots N \\
x \in R}}\left[\sum_{\alpha, \beta, \gamma=1}^{n} p^{(i) \alpha \beta} r_{\alpha \gamma} r_{\beta \gamma}\right], \\
& L=\max _{x \in R}\left[\sum_{\alpha, \beta, \gamma=1}^{n} r^{\alpha \beta} \operatorname{grad} r_{\alpha \gamma} \cdot \operatorname{grad} r_{\beta \gamma}\right],
\end{aligned}
$$

where we have written $p^{(i) \alpha \beta}$ for the inverse matrix of $p_{\alpha \beta}^{(i)}$, we have the analogue of (3.11)

$$
\begin{aligned}
h^{N} \sum_{R_{h}} \sum_{\alpha, \beta=1}^{n} \bar{r}_{\alpha \beta} v^{\alpha} v^{\beta} \geqq \int_{R} \sum_{\alpha, \beta=1}^{n} r_{\alpha \beta} u^{\alpha} u^{\beta} d V \\
\quad-\frac{h^{2}}{\pi^{2} r_{m}}\left[\left\{K \int_{R} \sum_{\alpha, \beta=1}^{n}\left(\sum_{i=1}^{n} p_{\alpha \beta}^{(i)} \frac{\partial u^{\alpha}}{\partial x^{i}} \frac{\partial u^{\beta}}{\partial x^{i}}+q_{\alpha \beta} u^{\alpha} u^{\beta}\right) d V\right\}^{1 / 2}\right. \\
\left.\quad+\left\{L \int_{R} \sum_{\alpha, \beta=1}^{n} r_{\alpha \beta} u^{\alpha} u^{\beta} d V\right\}^{1 / 2}\right]^{2}
\end{aligned}
$$

Similarly, defining ${ }^{3}$

$$
\bar{q}_{\alpha \beta}\left(m^{i} h\right) \equiv\left[h^{-N} \int_{0 \leqq \alpha^{i} \leqq h} q^{\alpha \beta}\left(m^{i} h+\alpha^{i}\right) d \alpha^{1} \cdots d \alpha^{N}\right]^{-1}
$$

where $q^{\alpha \beta}$ is the inverse matrix of $q_{\alpha \beta}$ and $^{3}$

$$
\bar{p}_{\alpha \beta}^{(i)}\left(m^{j} h\right) \equiv\left[h^{-N} \int_{0 \leqq \alpha^{j} \leqq h} p^{(i) \alpha \beta}\left(m^{j} h+\alpha^{j}\right) d \alpha^{1} \cdots d \alpha^{N}\right]^{-1},
$$

we find the analogues of (3.13) and (3.15). Thus, if we define the finite difference eigenvalues $\lambda_{1}^{(h)} \leqq \lambda_{2}^{(h)} \leqq \cdots$ as the successive minima of the ratio

$$
\frac{\sum_{\alpha, \beta=1}^{n}\left(\sum_{i=1}^{n} \bar{p}_{\alpha \beta}^{(i)} d_{i}\left[w^{\alpha}\right] d_{i}\left[w^{\beta}\right]+\bar{q}_{\alpha \beta} w^{\alpha} w^{\beta}\right)}{\sum_{\alpha, \beta=1}^{n} \bar{r}_{\alpha \beta} w^{\alpha} w^{\beta}}
$$

among sets of mesh functions $\left(w^{1}, \cdots, w^{n}\right)$ in class $M_{h}$, we find again the lower bound (3.27) for the eigenvalues of (5.1) in terms of those of (5.9). 


$$
\lambda_{k} \geqq \lambda_{k}^{(h)}\left[\frac{\left\{1+\frac{h^{2}}{\pi^{2} r_{m}}\left(K \lambda_{k}^{(h)}-L\right)\right\}^{1 / 2}-\frac{h^{2}}{\pi^{2} r_{m}}\left\{K L \lambda_{k}^{(h)}\right\}^{1 / 2}}{1+\frac{h^{2}}{\pi^{2} r_{m}} K \lambda_{k}^{(h)}}\right]^{2} .
$$

The considerations of $\S 4$ do not appear capable of extension to systems of elliptic differential equations containing mixed derivatives.

6. Error estimation. As has already been mentioned in the introduction, it is rather easy to get upper bounds for the eigenvalues $\lambda_{k}$ by means of another finite difference problem. Thus in order to determine the error, one must first calculate the eigenvalues of two finite difference problems. If the error turns out to be too large, one must reduce the mesh size and recalculate the eigenvalues. It is a great saving of labor to have an a priori estimate of the error in terms of the mesh size. For then one can pick a mesh size to give at most a given error and do only one eigenvalue computation.

We proceed to estimate the error by considering the scheme for obtaining upper bounds. For the sake of clarity we begin with the twodimensional Laplace operator case treated in $\S 2$.

Following a method suggested by $R$. Courant [2] (and already implicitly contained in a paper of L. Collatz [1]), we divide each square of the finite difference mesh into two triangles by means of a diagonal in a fixed direction. Then, given any mesh function $v$ of class $M_{h}$, we can associate with it a piecewise differentiable function $u$ by specifying that it coincides with $v$ at the mesh points, and is linear in each triangle. This function vanishes on the boundary of the domain $R_{h}$. Furthermore, if $v_{1}, \cdots, v_{k}$ are linearly independent mesh functions, the corresponding functions $u_{1}, \cdots, u_{k}$ are linearly independent; and to the linear combination $\xi_{1} v_{1}+\cdots+\xi_{k} v_{k}$ corresponds the linear combination $\xi_{1} u_{1}+\cdots+\xi_{k} u_{k}$. Letting $\mu_{k}\left(R_{h}\right)$ be the $k$ th eigenvalue of the fixed membrane problem

$$
\Delta u+\mu u=0 \quad \text { in } R_{h}
$$

with $u=0$ on the boundary of $R_{h}$, we have the Poincaré inequality

$$
\mu_{k}\left(R_{h}\right) \leqq \max _{\xi_{1}, \cdots, \xi_{k}} \frac{\int_{R_{h}}\left|\operatorname{grad}\left(\xi_{1} u_{1}+\cdots+\xi_{k} u_{k}\right)\right|^{2} d x d y}{\int_{R_{h}}\left(\xi_{1} u_{1}+\cdots+\xi_{k} u_{k}\right)^{2} d x d y} .
$$

Since $n$ depends linearly on its mesh values $v$, both the numerator and denominator in (6.2) are quadratic forms in the mesh function $v$. They have been explicitly determined by G. Polya [15], who finds that 


$$
\int_{R_{h}}|\operatorname{grad} u|^{2} d x d y=D_{h}(v)
$$

defined by (2.9), while

$$
\begin{aligned}
& \int_{R_{h}} u^{2} d x d y=I(v) \equiv h^{2} \sum_{R_{h}}\left\{v\left(x_{m}, y_{n}\right)^{2}-\frac{1}{12}\left[v\left(x_{m}+h, y_{n}\right)-v\left(x_{m}, y_{n}\right)\right]^{2}\right. \\
& \left.-\frac{1}{12}\left[v\left(x_{m}, y_{n}+h\right)-v\left(x_{m}, y_{n}\right)\right]-\frac{1}{12}\left[v\left(x_{m}+h, y_{n}+h\right)-v\left(x_{m}, y_{n}\right)\right]^{2}\right\} .
\end{aligned}
$$

We now let $\mu_{1}^{(h)} \leqq \mu_{2}^{(h)} \leqq \cdots$ be the successive minima of the ratio

$$
\frac{D_{h}(v)}{I(v)} .
$$

Letting $v_{1}, \cdots, v_{k}$ be the first $k$ minimizing functions, we see from (6.2) that

$$
\mu_{k}\left(R_{h}\right) \leqq \mu_{k}^{(h)} .
$$

Thus, we have upper bounds for the $\mu_{k}\left(R_{h}\right)$ in terms of the minimum problem (6.5), which can again be formulated as a finite difference problem. However, noting that

$$
I(v) \geqq h^{2} \sum_{R_{h}} v\left(x_{m}, y_{n}\right)^{2}-\frac{1}{4} h^{2} D_{h}(v),
$$

we can bound the $\mu_{k}^{(h)}$ in terms of the eigenvalues $\lambda_{k}^{(h)}$ by

$$
\mu_{k}^{(h)} \leqq \frac{\lambda_{k}^{(h)}}{1-\frac{1}{4} h^{2} \lambda_{k}^{(h)}},
$$

assuming, of course, that $h$ is so small that $h^{2} \lambda_{k}^{(h)}<4$. Thus, we have the upper bound

$$
\mu_{k}\left(R_{h}\right) \leqq \frac{\lambda_{k}^{(h)}}{1-\frac{1}{4} h^{2} \lambda_{k}^{(h)}} .
$$

This process is easily extended to $N$ dimensions. Here each mesh cube is divided in an arbitrary but fixed manner into simplices with vertices at the corners. Then the values of the mesh function $v$ determine a function $u$ coinciding with $v$ at the mesh points and linear in each simplex. We again find the bound (6.9) with the factor $1 / 4$ replaced by a constant $c_{N}$ depending on the dimensionality.

In the case of variable coefficients an extra error occurs because the coefficients appearing in the quadratic forms for the upper bound 
are different averages of the coefficients of the differential problem from those used in finding upper bounds. However, both are averages over cubes of size at most $2 h$. Thus the differences will be at most $h$ times a constant depending on the maximum gradients of the coefficients. This constant can be calculated. Thus we find in general

$$
\mu_{k}\left(R_{h}\right) \leqq \lambda_{k}^{(h)}+h f\left(h, \lambda_{k}^{(h)}\right)
$$

where $f\left(h, \lambda_{k}^{(h)}\right)$ is an explicitly known bounded non-decreasing function of $h$ and $\lambda_{k}^{(h)}$.

Now since $R$ is contained in $R_{h}, \mu_{k}\left(R_{h}\right)<\lambda_{k}$. However, if $R_{h}$ is close to $R$, we expect the $\mu_{k}\left(R_{h}\right)$ to be close to $\lambda_{k}$. The estimation of this closeness depends on the geometry of $R$. For example, if $R$ contains a cut, the domains $R_{h}$ will never have this cut, and so the $\mu_{k}\left(R_{h}\right)$ will not approach the $\lambda_{k}$. However, if $R$ is so smooth that the boundary of $R_{h}$ approaches that of $R$ as $h \rightarrow 0$, then it is easy to show that $\mu_{k}\left(R_{h}\right) \rightarrow \lambda_{k}$ and the inequality (6.10) together with the lower bound for $\lambda_{k}$ proves that $\lambda_{k}^{(h)} \rightarrow \lambda_{k}$.

If $R$ is convex and contains a circle of radius $\bar{r}$, then one can see that the image of $R$ under a dilatation of the ratio $\left(1+3 h \bar{r}^{-1}\right): 1$ about the center of this circle contains a region $R_{h}$. The eigenvalues of this image are $\left(1+3 h \bar{r}^{-1}\right)^{-2} \lambda_{k}$, and they now lie below the $\mu_{k}\left(R_{h}\right)$. Thus, using (6.10) we have

$$
\lambda_{k} \leqq\left(1+3 \overline{h \bar{r}^{-1}}\right)^{2}\left(\lambda_{k}^{(h)}+h f\left(h, \lambda_{k}^{(h)}\right)\right) .
$$

In other words, we have an upper bound for $\lambda_{k}$ differing from $\lambda_{k}^{(h)}$ by a term of order $h$. The difference between this and the lower bound thus approaches zero with $h$. In order to make this difference explicit, we need only bound $\lambda_{k}^{(h)}$ in terms of $\lambda_{k}$ by the inequality (3.26), (4.61), or (5.10) and use some upper bound for $\lambda_{k}$.

For another error estimate when $R$ is not convex the reader is referred to $\S 5$ of our previous paper [19]. While the argument is given there only for the lowest eigenvalue, it applies equally well to higher eigenvalues.

7. The non-homogeneous problem. We consider the elliptic differential equation

$$
\begin{array}{cc}
-\sum_{i, j=1}^{n} \frac{\partial}{\partial x^{i}}\left(a^{i j} \frac{\partial u}{\partial x^{j}}\right)+q u=G & \text { in } R, \\
u=0 & \text { on } \dot{R} .
\end{array}
$$

Here the coefficients $a^{i s}$ and $q$ satisfy the hypotheses of $\S 4$, and $G$ is a given continuous function. 
By the well-known Dirichlet principle, $u$ minimizes the ratio

$$
\frac{\int_{R}\left(\sum_{i, j=1}^{N} a^{i j} \frac{\partial \varphi}{\partial x^{i}} \frac{\partial \varphi}{\partial x^{j}}+q \varphi^{2}\right) d V}{\left(\int_{R} \varphi G d V\right)^{2}}
$$

among functions $\varphi$ vanishing on the boundary. Let the value of the minimum be $(1 / \lambda)$. It is easily seen from the equation (7.1) that

$$
\lambda=\int_{R} u G d V=\int_{R}\left(\sum_{i, j=1}^{N} a^{i j} \frac{\partial u}{\partial x^{i}} \frac{\partial u}{\partial x^{j}}+q u^{2}\right) d V .
$$

An upper bound for $(1 / \lambda)$ is easily found from the minimum principle. We proceed to find a lower bound. We define the mesh domain $R_{h}$ as before, the mesh function $v$ in terms of $u$ by (3.4), the mesh coefficients $\overline{a^{i j}}$ and $\bar{g}$ by (4.4) and (3.14), and the mesh function ${ }^{3}$

$$
\bar{G}\left(m^{i} h\right) \equiv h^{-N} \int_{0 \leqq \alpha^{i} \leqq h} G\left(m^{i} h+\alpha^{i}\right) d \alpha^{1} \cdots d \alpha^{N} .
$$

Then, by Schwarz's inequality, the free membrane problem for the cube, and (7.4)

$$
\begin{aligned}
& {\left[\int_{R} u G d V-h^{N} \sum_{R_{h}} v \bar{G}\right]^{2}} \\
& =\left[\sum_{R_{h}} \int_{0 \leqq \alpha^{i} \leqq h}\left\{u\left(m^{i} h+\alpha^{i}\right)-v\left(m^{i} h\right)\right\} G\left(m^{i} h+\alpha^{i}\right) d \alpha^{1} \cdots d \alpha^{N}\right]^{2} \\
& \quad \leqq\left[\sum_{R_{h}} \int_{0 \leqq \alpha^{i} \leqq h}\left\{u\left(m^{i} h+\alpha^{i}\right)-v\left(m^{i} h\right)\right\}^{2} d \alpha^{1} \cdots d \alpha^{N}\right] \\
& \quad \times\left[\sum_{R_{h}} \int_{0 \leqq \alpha^{i} \leqq h} G\left(m^{i} h+\alpha^{i}\right)^{2} d \alpha^{1} \cdots d \alpha^{N}\right] \\
& \quad \leqq \frac{h^{2}}{\pi^{2}} \int_{R}|\operatorname{grad} u|^{2} d V \int_{R} G^{2} d V \leqq \frac{h^{2} \lambda}{\pi^{2} A} \int_{R} G^{2} d V
\end{aligned}
$$

where

$$
A \equiv \min _{\eta_{1}, \ldots, \eta_{N}} \frac{\sum_{i, j=1}^{N} a^{i j} \eta_{i} \eta_{j}}{\eta_{1}^{2}+\cdots+\eta_{N}^{2}} .
$$

The inequality (7.5) gives the lower bound

$$
\left(h^{N} \sum_{R_{h}} v \bar{G}\right)^{2} \geqq\left[\lambda-\frac{h}{\pi}\left(\frac{\lambda}{A} \int G^{2} d V\right)^{1 / 2}\right]^{2} .
$$

We derive an upper bound for the form $Q(v)$ defined by (4.55) in 
the same way as we derived (4.58). We must, however, use the differential equation (7.1) instead of (4.1) and the single function $u$ instead of the linear combination (4.20). Thus, we find that the bound for the first integral on the right of (4.17) is, by inequality (4.23), just $c \lambda$ with $c$ defined by (4.22). However, the bound for the second integral on the right of (4.17) becomes (we again introduce the summation convention)

$$
P \lambda+2\left[\int_{R} a_{i j} f^{i} f^{j} G^{2} d V\right]^{1 / 2} \lambda^{1 / 2}
$$

instead of (4.24). Here we have defined

$$
P \equiv \max \left[2\left(q \alpha_{i j} f^{i} f^{j}\right)^{1 / 2}\right] .
$$

Thus, (4.26) is replaced by

$$
\oint_{\dot{R}} F\left(\frac{\rho u}{\partial n}\right)^{2} d S \leqq(c+P) \lambda+2\left(\int_{R} a_{i j} f^{i} f^{j} G^{2} d V\right)^{1 / 2} \lambda^{1 / 2} .
$$

Since $\mathscr{A}(u)$ does not necessarily vanish on the boundary, (4.36) becomes

$$
\begin{aligned}
& -\int_{R} a^{i j} \frac{\partial u}{\partial x^{i}} \frac{\partial}{\partial x^{j}}\left(\frac{\mathscr{A}(u)}{V g}\right) d V \\
& \quad=\int_{R} \frac{1}{V g} \mathscr{A}(u)^{2} d V-\oint_{\dot{R}} g^{i j} \frac{\partial u}{\partial x^{i}} n_{j} \mathscr{A}(u) d S .
\end{aligned}
$$

Using the differential equation and the triangle inequality we bound the first term on the right.

$$
\left\{\int_{R} \frac{1}{\sqrt{V} g} \mathscr{A}(u)^{2} d V\right\}^{1 / 2} \leqq\left(l_{3} \lambda\right)^{1 / 2}+\left(\int_{R} \frac{G^{2}}{V g} d V\right)^{1 / 2}
$$

where

$$
l_{3} \equiv \max (q / \sqrt{g}) .
$$

If we eliminate the derivatives of $\varphi$ between (4.43) and (4.44), without assuming $\mathscr{A}(u)$ to vanish on the boundary, we find

$$
\begin{aligned}
\frac{1}{2} a^{k l} & \frac{\partial}{\partial x^{k}}\left(a^{i j} \frac{\partial u}{\partial x^{i}} \frac{\partial u}{\partial x^{j}}\right) n_{\imath} \\
= & -\left[g^{p q} n_{p} n_{q}\right]^{3 / 2} \frac{\partial}{\partial x^{i}}\left(\left\{g^{k l} \frac{\partial R}{\partial x^{k}} \frac{\partial R}{\partial x^{l}}\right\}^{-(1 / 2)} \sqrt{g g^{i j}} \frac{\partial R}{\partial x^{j}}\right)\left(\frac{\partial u}{\partial n}\right)^{2} \\
& +g^{i j} \frac{\partial u}{\partial x^{i}} n_{j} \mathscr{A}(u) .
\end{aligned}
$$

The integral of the second term just cancels the boundary terms of (7.11) when we substitute in (4.35). The first terms on the right of (7.14) is bounded as before by $e \oint F(\partial u / \partial n)^{2} d S$ where $e$ is defined by (4.47). 
Inequality (4.41) remains unchanged. Thus we derive in the same way as (4.58) that

$$
\begin{aligned}
& \{Q(v)\}^{1 / 2} \leqq \lambda^{1 / 2}+h\left\{\frac{11}{30} a b\right\}^{1 / 2}\left\{\left(c ə+P e+l_{3}+d\right) \lambda\right. \\
& \left.+2\left[e\left(\int_{R} a_{i j} f^{i} f^{j} G^{2} d V\right)^{1 / 2}+\left(l_{3} \int_{R} \frac{G^{2}}{V g} d V\right)^{1 / 2}\right] \lambda^{1 / 2}+\int_{R} \frac{G^{2}}{V g} d V\right\}^{1 / 2} \\
& +h\left\{\frac{11}{30} a m \lambda^{\}^{1 / 2}}+h^{1 / 2}\left\{\frac{N \nu_{h} a}{2 F_{m}}\right\}^{1 / 2}\{(c+P) \lambda\right. \\
& \left.+2\left(\int_{R} a_{i j} f^{i} f^{j} G^{2} d V\right)^{1 / 2} \lambda^{1 / 2}\right\}^{1 / 2} .
\end{aligned}
$$

We now define

$$
\frac{1}{\lambda^{(h)}} \equiv \min _{w \in M_{h}} \frac{Q(w)}{\left(h^{N} \sum_{R_{h}} w \bar{G}\right)^{2}} .
$$

This quantity may be computed by a finite difference analogue of (7.1). By the minimum property,

$$
\frac{1}{\lambda^{(h)}} \leqq \frac{Q(v)}{\left(h^{N} \sum_{R_{h}} v \bar{G}\right)^{2}} .
$$

But the right-hand side is bounded by an explicit function of $\lambda$ and $h$ of the form $(1 / \lambda)+o\left(h^{1 / 2}\right)$ by means of (7.7) and (7.15). This gives a lower bound for $1 / \lambda$ in terms of $1 / \lambda(h)$.

The absence of mixed derivatives results in a great simplification. Inequality (3.19) is valid, and we find

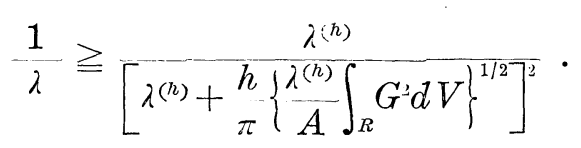

The upper bound for $1 / \lambda$ can again be obtained by means of a finite difference method using piecewise linear functions. Once this piecewise linear function and the error (difference between upper and lower bounds) is known, one can find a pointwise approximation to $u$ at any interior point by the method of Diaz and Greenberg [3, 4].

8. Higher order operators. The methods of $\S 3$ are easily extended to the eigenvalue problem

$$
L u=\lambda r u \quad \text { in } R
$$

where $L$ is an elliptic operator of order $2 m$, and all derivatives of orders 
up to $m-1$ of $u$ vanish on $\dot{R}$, provided the numerator of the corresponding Rayleigh quotient is the integral of a linear combination of squares of derivatives of $u$.

We illustrate the extension by applying it to the problem of the vibrating clamped plate

$$
\begin{array}{ll}
\Delta \Delta u=\lambda u & \text { in } R, \\
u=\partial u / \partial n=0 & \text { on } \dot{R}
\end{array}
$$

in two dimensions. The Rayleigh quotient may be written as

$$
\frac{\iint_{R}\left[\left(\frac{\partial^{2} u}{\partial x^{2}}\right)^{2}+2\left(\frac{\partial^{2} u}{\partial x \partial y}\right)^{2}+\left(\frac{\partial^{2} u}{\partial y^{2}}\right)^{2}\right] d x d y}{\iint_{R} u^{2} d x d y}
$$

The domain $R_{h}$ is defined as before. $M_{h}$ is the set of mesh functions vanishing everywhere except at the interior mesh points of $R_{h}$. The finite difference eigenvalues $\lambda_{k}^{(h)}$ are defined as the successive minima of the ratio

$$
\frac{Q(w)}{h^{2} \sum_{R_{h}} w^{2}}
$$

with $w \in M_{h}$ and

$$
\begin{gathered}
h^{2} Q(w) \equiv \sum_{R_{h}}\left\{[w(m h+h, n h)-2 w(m h, n h)+w(m h-h, n h)]^{2}\right. \\
+2[w(m h+h, n h+h)-w(m h+h, n h) \\
-w(m h, n h+h)+w(m h, n h)]^{2} \\
\left.+[w(m h, n h+h)-2 w(m h, n h)+w(m h, n h-h)]^{2}\right\} .
\end{gathered}
$$

The mesh function $v$ is related by means of (2.10) to the function $u$ having continuous first derivatives and piecewise continuous second derivatives and vanishing outside $R$.

We now find

$$
\begin{gathered}
\text { (8.6) } \iint_{R}\left(\frac{\partial^{2} u}{\partial x^{2}}\right) d x d y-h^{-2} \sum_{R_{n}}[v(m h+h, n h)-2 v(m h, n h)+v(m h-h, n h)]^{2} \\
=h^{-2} \sum_{R_{h}} \int_{-h}^{2 h} d \alpha \int_{0}^{h} d \beta \tilde{\psi}(\alpha)\left[\frac{\partial^{2} u}{\partial x^{2}}(m h+\alpha, n h+\beta)-h^{-2}\{v(m h+h, n h)\right. \\
-2 v(m h, n h)+v(m h-h, n h)\}]^{2} \geqq 0 .
\end{gathered}
$$

We have put 


$$
2 \tilde{\psi}(\alpha)= \begin{cases}(\alpha+h)^{2} & -h \leqq \alpha \leqq 0 \\ h^{2}+2 h \alpha-2 \alpha^{2} & 0 \leqq \alpha \leqq h \\ (2 h-\alpha)^{2} & h \leqq \alpha \leqq 2 h\end{cases}
$$

A similar inequality holds for $\partial^{2} u / \partial y^{2}$. For the mixed derivative we have

$$
\begin{aligned}
& \iint_{R}\left(\frac{\partial^{2} u}{\partial x \partial y}\right)^{2} d x d y-h^{-2} \sum_{R_{h}} \\
\times & {[v(m h+h, n h+h)-v(m h+h, n h)-v(m h, n h+h)+v(m h, n h)]^{2} } \\
= & h^{-2} \sum_{R_{h}} \int_{0}^{2 h} \int_{0}^{2 h} \psi(\alpha) \psi(\beta) \\
\times & {\left[\frac{\partial^{2} u}{\partial x \partial y}(m h+\alpha, n h+\beta)-h^{-2}\{v(m h+h, n h+h)-v(m h+h, n h)\right.} \\
- & v(m h, n h+h)+v(m h, n h)\}]^{2} d \alpha d \beta \\
\geqq & 0
\end{aligned}
$$

with $\psi(\alpha)$ defined by (2.20).

Thus $Q(v)$ is bounded by the numerator of (8.3). For the denominator of (8.4) we use the inequality (2.14) together with Green's theorem and Schwarz's inequality to give

$$
\iint_{R} u^{2} d x d y-h^{2} \sum_{R_{h}} v^{2} \leqq \frac{h^{2}}{\pi^{2}}\left|\iint_{R} u^{2} d x d y \iint_{R} u \Delta \Delta u d x d y\right|^{1 / 2}
$$

The substitution (2.15) and Poincare's inequality then give

$$
\lambda_{k}^{(h)} \leqq \frac{\lambda_{k}}{1-\left(h^{2} / \pi^{2}\right) \lambda_{k}^{1 / 2}},
$$

which is a lower bound for $\lambda_{k}$.

\section{BIBLIOGRAPHY}

1. L. Collatz, Konvergenz des Differenzverfahrens bei Eigenwertproblemen partieller Differentialgleichungen, Deutsche Math. 3 (1938), 200-212.

2. R. Courant, Variational methods for the solutions of problems of equilibrium and vibrations, Bull. Amer. Math. Soc. 49 (1943), 1-23.

3. J. B. Diaz and H. J. Greenberg, Upper and lower bounds for the solution of the first biharmonic boundary value problem, J. Math. Phys. 27 (1948), 193-201.

4. H. J. Greenberg, The determination of upper and lower bounds for the solution of the Dirichlet problem, J. Math. Phys. 27 (1948), 161-182.

5. G. E. Forsythe, Asymptotic lower bounds for the fundamental frequency of certain polygonal membranes, Pacific J. Math. 4 (1954), 467-480.

6. G. E. Forsythe, Asymptotic lower bounds for the fundamental frequency of convex membranes, Pacific J. Math. 5 (1955), 691-702.

7. G. E. Forsythe, Difference methods on a digital computer for Laplacian boundary 
value and eigenvalue problems, Comm. Pure Appl. Math. 9 (1956), 425-434.

8. J. Hersch, Équations différentielles et fonctions des cellules, C. R. Acad. Sci. Paris 240 (1955), 1602-1604.

9. L. Hörmander, Uniqueness theorems and estimates for normally hyperbolic partial differential equations of second order, Tolfte Skandinavska Matematikerkongressen Lund, 1953, 105-115.

10. L. E. Payne, Inequalities for eigenvalues of membranes and plates, J. Rat. Mech. 4 (1955), 517-529.

11. L. E. Payne and H. F. Weinberger, New bounds in harmonic and biharmonic problems, J. Math. Phys. 33 (1955), 291-307.

12. L. E. Payne and H. F. Weinberger, A generalized Rellich identity, Bull. Amer. Math. Soc. 63 (1957), 121.

13. L. E. Payne and H. F. Weinberger, New bounds for solutions of second order elliptic partial differential equations. University of Maryland Technical Note BN-108, AFOSRTN-57-651 AD-136 636 (1957). To appear in Pacific J. Math.

14. H. Poincaré, Sur les équations aux dérivées partielles de la physique mathématique. Amer. J. Math. 12 (1890), 211-294.

15. G. Polya, Sur une interprétation de la méthode des différences finies qui peut fournir des bornes supérieures ou inférieures, C. R. Acad. Sci. Paris 235 (1952), 995-997.

16. F. Rellich, Darstellung der Eigenwerte von $\Delta u+\lambda u$ durch ein Randintegral, Math. $Z$. 46 (1940), 635-646.

17. J. L. Synge and A. Schild, Tensor Calculus. Toronto Press, 1949.

18. H. F. Weinberger, Lower bounds for minima by finite difference methods, Bull. Amer. Math. Soc. 61 (1955), 301.

19. H. F. Weinberger, Upper and lower bounds for eigenvalues by finite difference methods, Comm. Pure Appl. Math. 9 (1956), 613-623. (Also Proceedings of the Conference on Partial Differential Equations, Berkeley, 1955.)

20. A. Weinstein, Étude des spectres des équations aux dérivées partielles de la théorie des plaques élastiques, Mém. Sci. Math. 88 (1937).

Mathematics Research Center, University of Wisconsin 


\section{PACIFIC JOURNAL OF MATHEMATICS}

\section{EDITORS}

\section{H. L. Royden}

Stanford University

Stanford, California

\section{R. A. Beaumont}

University of Washington

Seattle 5 , Washington

\author{
A. L. Whiteman
}

University of Southern California

Los Angeles 7, California

E. G. Straus

University of California

Los Angeles 24, California

\section{ASSOCIATE EDITORS}
E. F. BECKENBACH
A. HORN
L. NACHBIN
G. SZEKERES
C. E. BURGESS
V. GANAPATHY IYER
I. NIVEN
F. WOLF
M. HALL
R. D. JAMES
T. G. OSTROM
E. HEWITT
M. S. KNEBELMAN
M. M. SCHIFFER
K. YOSIDA

\section{SUPPORTING INSTITUTIONS}

\author{
UNIVERSITY OF BRITISH COLUMBIA \\ CALIFORNIA INSTITUTE OF TECHNOLOGY \\ UNIVERSITY OF CALIFORNIA \\ MONTANA STATE UNIVERSITY \\ UNIVERSITY OF NEVADA \\ OREGON STATE COLLEGE \\ UNIVERSITY OF OREGON \\ UNIVERSITY OF SOUTHERN CALIFORNIA
}

\author{
STANFORD UNIVERSITY \\ UNIVERSITY OF UTAH \\ WASHINGTON STATE COLLEGE \\ UNIVERSITY OF WASHINGTON \\ * * * * \\ AMERICAN MATHEMATICAL SOCIETY \\ CALIFORNIA RESEARCH CORPORATION \\ HUGHES AIRCRAFT COMPANY \\ THE RAMO-WOOLDRIDGE CORPORATION
}

Mathematical papers intended for publication in the Pacific Journal of Mathematics should be typewritten (double spaced), and the author should keep a complete copy. Manuscripts may be sent to any of the editors. All other communications to the editors should be addressed to the managing editor, E. G. Straus at the University of California, Los Angeles 24, California.

50 reprints per author of each article are furnished free of charge; additional copies may be obtained at cost in multiples of 50 .

The Pacific Journal of Mathematics is published quarterly, in March, June, September, and December. The price per volume (4 numbers) is $\$ 12.00$; single issues, $\$ 3.50$. Back numbers are available. Special price to individual faculty members of supporting institutions and to individual members of the American Mathematical Society: $\$ 4.00$ per volume; single issues, $\$ 1.25$.

Subscriptions, orders for back numbers, and changes of address should be sent to Pacific Journal of Mathematics, 2120 Oxford Street, Berkeley 4, California.

Printed at Kokusai Bunken Insatsusha (International Academic Printing Co., I.td.), No. 10, 1-chome, Fujimi-cho, Chiyoda-ku, Tokyo, Japan.

PUBLISHED BY PACIFIC JOURNAL OF MATHEMATICS, A NON-PROFIT CORPORATION

The Supporting Institutions listed above contribute to the cost of publication of this Journal, but they are not owners or publishers and have no responsibility for its content or policies. 


\section{Pacific Journal of Mathematics}

\section{Vol. 8, No. 2 \\ April, 1958}

John Herbert Barrett, Second order complex differential equations with a real independent variable ............................ 187

Avner Friedman, Remarks on the maximum principle for parabolic equations and its applications ......................... 201

Richard Robinson Goldberg, An inversion of the Stieltjes transform ....... 213

Olavi Hellman, On the periodicity of the solution of a certain nonlinear integral equation .................................. 219

Gilbert Helmberg, A theorem on equidistribution on compact groups...... 227

Lloyd Kenneth Jackson, Subfunctions and the Dirichlet problem ......... 243

Naoki Kimura, The structure of idempotent semigroups. I ............ 257

Stephen Kulik, A method of approximating the complex roots of equations........................................ 277

Ancel Clyde Mewborn, A note on a paper of L. Guttman.............. 283

Zeev Nehari, On the principal frequency of a membrane ............ 285

G. Pólya and I. J. Schoenberg, Remarks on de la Vallée Poussin means and convex conformal maps of the circle ...................... 295

B. M. Stewart, Asymmetry of a plane convex set with respect to its centroid .......................................... 335

Hans F. Weinberger, Lower bounds for higher eigenvalues by finite difference methods

Edwin Weiss and Neal Zierler, Locally compact division rings ......... 369

Bertram Yood, Homomorphisms on normed algebras ................. 373 